\section{(A) Check for updates}

Cite this: Food Funct., 2021, 12, 2489

\title{
Lactobacillus plantarum and Bifidobacterium bifidum alleviate dry eye in mice with exorbital lacrimal gland excision by modulating gut inflammation and microbiota $\dagger$
}

\author{
Soo-won Yun, $\star^{\mathrm{a}}$ Young-Hoo Son, $t^{\mathrm{b}}$ Dong-Yun Lee, ${ }^{\mathrm{b}}$ Yoon-Jung Shin, ${ }^{\mathrm{b}}$ \\ Myung Joo Han ${ }^{\star a}$ and Dong-Hyun Kim (D) *b
}

\begin{abstract}
In order to understand the efficacy of probiotics against dry eye syndrome, we selected anti-inflammatory probiotics Lactobacillus plantarum NK151 and Bifidobacterium bifidum NK175, which increased the ratio of IL-10 to TNF- $\alpha$ expression, from the human gut bacteria collection and examined their effects on tear secretion and cornea/conjunctiva inflammation in mice with excision of the unilateral exorbital lacrimal gland and $1 \%$ atropine and $0.1 \%$ benzalkonium chloride solution (ELA)-induced dry eye. Exposure to ELA significantly reduced tear secretion in mice, assessed by the phenol red thread tear test. However, oral gavage of NK151 and/or NK175 significantly increased ELA-suppressed tear secretion, IL-10 expression, and goblet cell population and decreased the ELA-induced corneal fluorescein-staining score, IL-1 $\beta$ and TNF- $\alpha$ expression in the conjunctiva. They also suppressed ELA-induced myeloperoxidase, IL-1 $\beta$, and TNF- $\alpha$ expression. In particular, they increased the ratio of IL-10 to TNF- $\alpha$ expression in the colon. Their treatments increased ELA-induced $\alpha$-diversity reduction to that of the control group and partially restored ELA-shifted $\beta$-diversity to that of the control group. Oral gavage of NK151 and/or NK175 reduced ELAinduced Verrucomicrobia and Actinobacteria populations at the phylum level. Furthermore, they reduced ELA-induced Bacteroidaceae, Akkemansiaceae, and AC160630_f populations and increased ELA-suppressed Lactobacillaceae and Muribaculaceae populations at the family level. These gut bacteria populations exhibited significant correlation with the tear secretion volume. In conclusion, NK151 and/or NK175 alleviated dry eye by modulating the expression ratio of pro-inflammatory cytokines such as TNF- $\alpha$ and anti-inflammatory cytokines such as IL-10 and gut microbiota composition.
\end{abstract}

Received 12th November 2020 Accepted 1st February 2021

DOI: $10.1039 /$ dOfo02984j

rsc.li/food-function

\footnotetext{
${ }^{a}$ Department of Food and Nutrition, Kyung Hee University, 26, Kyungheedae-ro, Dongdaemun-gu, Seoul 02447, Korea.E-mail: mjhan@khu.ac.kr; Fax: +82-2-961-0260

${ }^{b}$ Neurobiota Research Center, College of Pharmacy, Kyung Hee University, 26, Kyungheedae-ro, Dongdaemun-gu, Seoul 02447, Korea.E-mail: dhkim@khu.ac.kr; Fax: $+82-2-957-5030$

$\dagger$ Electronic supplementary information (ESI) available: Table S1. Ingredients of the mouse chow diet used in the present study. Table S2. Food intake and weight gain of mice. Table S3. Effects of NK151, NK175, and NKM on the gut microbiota composition at the phylum level in mice with ELA-induced dry eye. Table S4. Effects of NK151, NK175, and NKM on the gut microbiota composition at the family level in mice with ELA-induced dry eye. Table S5. Effects of NK151, NK175, and NKM on the gut microbiota composition at the genus level in mice with ELA-induced dry eye. Table S6. Effects of NK151, NK175, and NKM on the gut microbiota composition at the species level in mice with ELA-induced dry eye. Fig. S1. Effects of five gut bacteria isolated from the human fecal bacteria collection on the LPS-induced TNF- $\alpha$ and IL-10 expression in macrophage cells. Fig. S2. Effect of NK151 on the ELA-induced dry eye in C57BL/6 mice. Fig. S3. The mixture of NK151 and NK175 (4:1), NKm, alleviated ELA-induced colitis in BALB/c mice. See DOI: 10.1039/d0fo02984j

$\$$ Equally contributed.
}

\section{Introduction}

Dry eye disease (DED) is the most common eye disease, accounting for $>5 \%$ of the world's population. ${ }^{1}$ DED can cause ocular discomfort and visual impairment. ${ }^{2,3}$ Currently, the occurrence of DED is significantly increasing due to the popularization of air conditioning, increased wearers of soft contact lenses, and frequent usage of visual display terminals such as smartphones and personal computers. ${ }^{3-5}$ Dry eye increases the expression of inflammation factors such as tumor necrosis factor (TNF)- $\alpha$ and interleukin (IL)- $1 \beta$ in the ocular surface, which causes damage of corneal epithelial cells and loss of conjunctival goblet cells. ${ }^{6,7}$ Long-term dry eye progresses to visual disturbance and tear film instability. ${ }^{8}$ To treat patients with DED, artificial tears, which contain hyaluronic acid, cyclosporine A, and tetracyclines, are available. ${ }^{9}$ Besides these, other agents including anti-inflammatory components have been developed. ${ }^{10,11}$ However, the long-term application of these medications sometimes leads to adverse drug reactions, 
such as ocular hyperemia, corneal calcification, ocular hypertension, glaucoma, cataract, and infection.

The gut microbiota are involved in the development and functioning of basic physiological processes of the host. ${ }^{12,13}$ Therefore, they are closely associated with the occurrence of multiple chronic diseases, such as obesity, psychiatric disorder, and chronic inflammatory diseases. ${ }^{14,15}$ Recently, a series of studies have highlighted the influence of the gut microbiota on DED including Sjögren's syndrome. ${ }^{16-18}$ Moon et al. reported that the feces of patients with Sjögren's syndrome had a higher abundance of Bacteroidetes and a lower abundance of Bifidobacterium compared to those of healthy individuals. ${ }^{19}$ They also reported that patients with DED had increased Veillonella and reduced Subdoligranulum compared to healthy individuals. de Paiva et al. reported that exposure to desiccating stress/antibiotics caused Sjögren's syndrome-like dry eye in mice. ${ }^{20}$ Their exposure leads to a decrease in Clostridium and an increase in Enterobacter, Escherichial Shigella, and Pseudomonas in the feces. The feces of patients with Sjögren's syndrome have a greater relative abundance of Pseudobutyrivibrio, Escherichia/Shigella, Blautia, and Streptococcus and a lower abundance of Bacteroides, Parabacteroides, Faecalibacterium, and Prevotella than those of healthy individuals. However, fecal microbiota transplantation from the control mice alleviates DED in the transplanted germ-free CD25 KO mice with Sjögren's syndrome-like dry eye. ${ }^{21}$ The probiotic IRT-5 also alleviates the ocular staining score and IL-1 $\beta$ and IL-10 expression and increases Lactobacillus populations in NOD.B10.H2b mice, a potential model for primary Sjögren's syndrome. ${ }^{22}$ Oral administration of a Bifidobacterial mixture with fructooligosaccharide (a symbiotic combination) alleviates dry eye in patients with DED. ${ }^{23}$ These results suggest that the gut microbiota and their byproducts such as endotoxins and short chain fatty acids (SCFAs) may be associated with the occurrence of DED. Therefore, regulating the gut microbiota may be beneficial for the therapy of DED.

In the present study, to understand the efficacy of probiotics against DED, we selected anti-inflammatory probiotics NK151 and NK175, which increased the ratio of IL-10 to TNF- $\alpha$ expression, from the human gut bacteria collection and examined their therapeutic effects on dry eye, colitis, and gut microbiota composition in mice with exorbital lacrimal gland excision and $1 \%$ atropine $/ 0.1 \%$ benzalkonium chloride treatment (ELA)-induced dry eye.

\section{Materials and methods}

\subsection{Materials}

Fluorescein sodium salt, lissamine green, LPS, 4',6-diamidino2-phenylindole dilactate (DAPI), and sodium thioglycolate were purchased from Sigma (St Louis, MO). The antibody for TNF- $\alpha$ was purchased from Santa Cruz Technology (Santa Cruz, CA). Enzyme-linked immunosorbent assay (ELISA) kits for IL-1 $\beta$, TNF- $\alpha$, and myeloperoxidase were purchased from R\&D
Systems (Minneapolis, MN). The ELISA kit for IL-10 and Alexa Fluor 488 were purchased from Invitrogen (Waltham, MA). Phenol red-impregnated cotton threads were purchased from Zone Quick (Showa Yakuhin Kako Co., Ltd, Tokyo, Japan). De Man, Rogosa and Sharpe (MRS) medium was purchased from BD (Franklin Lakes, NJ). The QIAamp Fast DNA stool mini kit was purchased from Qiagen (Hilden, Germany).

\subsection{Animals}

C57BL/6 mice (male, 18-21 g, 5 weeks old) and BALB/c mice (male, 19-21 g, 7 weeks old) were obtained from Koatech (Seongnam-Shi, Korea) and acclimated for 1 week before experiments. Mice were housed in plastic cages with a raised wire floor at $20-22{ }^{\circ} \mathrm{C}$ and $50 \pm 10 \%$ humidity under a $12 \mathrm{~h}$ light/ dark cycle (artificial light, 150 lux from 8 am to $8 \mathrm{pm}$ ), and fed with water and a chow diet (ESI Table S1†) ad libitum. All animal experiments were approved by the Institutional Animal Care and Use Committee of Kyung Hee University (IACUC no. KHSASP-19-199) and performed in accordance with the NIH and University Guide for Laboratory Animal Care and Usage.

\subsection{Culture of Lactobacillus plantarum NK151 and Bifidobacterium bifidum NK175 and their dosage regimen}

Gut bacteria including NK151 and NK175, which suppressed IL-1 $\beta$ expression in activated macrophages, were cultured in general media for probiotics such as De Man, Rogosa and Sharpe (MRS) broth, centrifuged at $5000 \mathrm{~g}$ and $4{ }^{\circ} \mathrm{C}$ for $20 \mathrm{~min}$, and washed with saline. The collected cells were suspended in saline and tyndallized by heating at $90{ }^{\circ} \mathrm{C}$ for $30 \mathrm{~min}$ twice, as previously reported. ${ }^{24}$ For the in vitro and in vivo experiments, the collected cells were freeze-dried and suspended in saline.

\subsection{Culture of peritoneal macrophages}

For anti-inflammatory probiotics in activated macrophages, TNF- $\alpha$ expression-suppressing NK151 and NK175, which did not exhibit hemolytic activity in 7\% v/v sheep blood-containing agar plates, were selected from the human gut bacterial strain collection in LPS-stimulated macrophages. Briefly, macrophage cells, which were isolated from the peritoneal cavity of mice according to the method of Jang et al. ${ }^{24}$ were suspended in RPMI1640 containing 1\% antibiotics and 10\% fetal bovine serum, seeded in a 6-well plate and incubated with LPS (100 $\left.\mathrm{ng} \mathrm{mL}^{-1}\right)$ in the absence or presence of gut bacteria $\left(1 \times 10^{5}\right.$ $\mathrm{CFU} \mathrm{mL}^{-1}$ ) for $20 \mathrm{~h}$ (ESI Fig. S1 $\dagger$ ). TNF- $\alpha$, IL-1 $\beta$, and IL-10 levels were assayed in the cultured solution using ELISA kits.

\subsection{Dosage regimen of Lactobacillus plantarum NK151 and Bifidobacterium bifidum NK175}

To decide the dosage of NK151 and NK175 in the in vivo experiment, NK151 at dosages of $5 \times 10^{8}$ and $1 \times 10^{9}$ colonyforming units (CFU) per mouse per day was orally gavaged once a day for 10 days in mice with ELA-induced dry eye. NK151 at dosages of $5 \times 10^{8}$ and $1 \times 10^{9}$ CFU per mouse per day increased ELA-suppressed tear secretion (ESI Fig. S2 $\dagger$ ). Therefore, we orally treated NK151 and/or NK175 at a dose of 5 $\times 10^{8} \mathrm{CFU}$ per mouse per day in the in vivo experiments. 


\subsection{Preparation of mice with dry eye}

To prepare mice with ELA-induced dry eye, mice were anesthetized with $2 \%$ isoflurane and maintained with $1 \%$ isoflurane and the mouse right exorbital lacrimal gland was excised, as reported previously. ${ }^{25}$ Thereafter, one drop of $1 \%$ atropine/ $0.1 \%$ benzalkonium chloride solution was added to the cornea of the right eye twice a day for 5 days. Sham mice (SHA) were operated without the right exorbital lacrimal gland excision and not treated with $1 \%$ atropine/ $0.1 \%$ benzalkonium chloride solution.

First, C57BL/6 mice were randomly divided into six groups: (1) the CON group treated with the vehicle ( $0.1 \mathrm{~mL}$ of saline) in control mice, (2) the SHA group treated with the vehicle in sham mice, (3) the DE group treated with the vehicle in mice exposed to ELA, (4) the LP group treated with NK151 $\left(5 \times 10^{8}\right.$ CFU per mouse per day, suspended in $0.1 \mathrm{~mL}$ of saline) in mice exposed to ELA, (5) the BB group treated with NK175 (5 $\times$ $10^{8} \mathrm{CFU}$ per mouse per day, suspended in $0.1 \mathrm{~mL}$ of saline) in mice exposed to ELA, and (6) the group treated with the NK151 and NK175 (3:2) mixture, NKM, $\left(5 \times 10^{8}\right.$ CFU per mouse per day, suspended in $0.1 \mathrm{~mL}$ of saline) in mice exposed to ELA. Second, BALB/c mice were randomly divided into three groups: (7) the SHA group treated with the vehicle in sham mice, (8) the DE group treated with the vehicle in mice exposed to ELA, and (9) the group treated with the NK151 and NK175 (4:1) mixture, NKm, $\left(5 \times 10^{5} \mathrm{CFU}\right.$ per mouse per day, suspended in $0.1 \mathrm{~mL}$ of saline) in mice exposed to ELA.

Mice were sacrificed by $\mathrm{CO}_{2}$ inhalation. Eyes with ocular adnexa and colons were collected and stored at $-80^{\circ} \mathrm{C}$ for the assay of biochemical markers. For the immunohistochemistry assay, mice were transcardiacally perfused with $4 \%$ paraformaldehyde for eye tissue fixation. Eye tissues with the conjunctiva were post-fixed with $4 \%$ paraformaldehyde for $4 \mathrm{~h}$, cytoprotected in $30 \%$ sucrose solution, frozen, and cut using a cryostat.

\subsection{Tear volume measurement}

The tear volume was measured at day 10 after the treatment with ELA. $^{26}$ Phenol red-impregnated cotton threads were placed in the lateral canthus for $30 \mathrm{~s}$. The tear volume was indicated as the length $(\mathrm{cm})$ of the color-changed thread that absorbed the tear fluid.

\subsection{Eye-blink counting}

The faces of mice with or without ELA-induced dry eye were recorded for 3 min using a digital camera and the number of eye blinks was counted. ${ }^{27}$

\subsection{Corneal fluorescein staining}

To examine corneal epithelial damage, corneal fluorescein staining was performed, as reported previously. ${ }^{28}$ One microliter of $2 \%$ fluorescein was dropped into the lateral conjunctival sac and the corneal fluorescein-staining score $(0$, none; 1 , mild; 2, moderate; and 3, severe) was counted in four different corneal areas (upper right, upper left, lower right, and lower left areas of the cornea) 10 min after fluorescein treatment, as reported previously. ${ }^{28}$

\subsection{Conjunctival lissamine green staining}

To evaluate conjunctival damage, lissamine green staining was performed, as reported previously. ${ }^{29}$ One microliter of $20 \%$ lissamine green solution was dropped into the lateral conjunctiva sac and the grade scale (0-4) for conjunctival damage was scored.

\subsection{Evaluation of meibomian lacrimal gland dysfunction}

We observed the morphological changes in the eyelid margin and meibomian gland orifices, as reported previously. ${ }^{30}$ The dysfunction of meibomian glands including abnormalities of lid margins and expression of meibum was scored from 0 to $3 .^{31}$

\subsection{ELISA}

Eye and colon tissues were homogenized with ice-cold radioimmunoprecipitation assay lysis buffer containing a $1 \%$ phosphatase inhibitor cocktail and a $1 \%$ protease inhibitor cocktail and centrifuged at $10000 \mathrm{~g}$ and $4{ }^{\circ} \mathrm{C}$ for $20 \mathrm{~min} .{ }^{24}$ The resulting supernatants were transferred to a 96-well plate. Cytokine (TNF- $\alpha$, IL-1 $\beta$, and IL-10) and myeloperoxidase levels were assayed using ELISA kits.

\subsection{Immunofluorescence assay}

Immunofluorescence assay was performed according to the method of Kim et al. ${ }^{32}$ Briefly, the sections of the eye with the conjunctiva were washed with phosphate-buffered saline, blocked with normal serum, incubated with antibodies for TNF- $\alpha$ (1:200), NF-кB (1:100), and CD11c (1:200) overnight, and treated with the secondary antibody for $2 \mathrm{~h}$. The secondary antibody conjugated with Alexa Fluor 488 (1:200) or Alexa Fluor 594 (1:200) was then applied to visualize. The nuclei were stained with DAPI.

\subsection{S rRNA gene pyrosequencing}

Bacterial 16S rRNA gene pyrosequencing was performed according to the method of Kim et al. ${ }^{32}$ Genomic DNA was extracted from fresh stools of mice using the QIAamp DNA stool mini kit. Genomic DNA amplification was carried out using barcoded primers, which targeted the $\mathrm{V} 4$ region of a bacterial 16S rRNA gene. Each amplicon sequencing was performed using Illumina iSeq 100 (San Diego, CA, USA). Pyrosequencing reads were deposited in the NCBI's short read archive under accession number PRJNA674909.

\subsection{Statistical analysis}

All experimental data are given as mean \pm standard deviation (SD) and analyzed using GraphPad Prism 8 (GraphPad Software Inc., San Diego, CA). The significance was analyzed by using the Kruskal-Wallis test with Dunn's post-hoc test for non-parametric analysis $(p<0.05)$. 


\section{Results}

3.1. NK151 and NK175 increased the ratio of IL-10 to TNF- $\alpha$ expression in LPS-stimulated macrophages

We selected IL-10 expression-inducing probiotics NK151 and NK175 from the human fecal bacteria strain collection. They potently induced IL-10 expression in LPS-stimulated macrophages, while the TNF- $\alpha$ and IL- $1 \beta$ expression was suppressed (Fig. 1A-C). In particular, NK151 and NK175 also increased the ratio of IL-10 to TNF- $\alpha$ expression (Fig. 1D). NK151 and NK175 were identified as Lactobacillus plantarum and Bifidobacterium bifidum on the basis of the results of Gram staining, 16S rRNA gene sequencing, and API 50 CHL kit assay.

\subsection{NK151, NK175, and NKM alleviated ELA-induced dry eye} in mice

We examined the effects of NK151, NK175, and their $(3: 2)$ mixture, NKM, on tear secretion in C57BL/6 mice with ELAinduced dry eye using the phenol red thread tear test (Fig. 2). Exposure to ELA in mice for 5 days significantly reduced tear secretion to $18.4 \%$ of sham mice on day 10 after the final exposure to ELA $(p<0.05)$ (Fig. 2A and ESI Table S2 $\dagger)$. The tear secretion volume of sham mice was not significantly different from that of control mice. However, oral gavage of NK151, NK175, or NKM significantly increased tear secretion $6 \mathrm{~h}$ after the final gavage of probiotics: their treatments increased the tear volume to $33.4 \%, 28.4 \%$, and $31.3 \%$ of sham mice, respectively. Exposure to ELA significantly increased the corneal fluorescein-staining score in mice to $3.7 \pm 2.4$ (Fig. 2B). However, oral gavage of NK151, NK175, or NKM decreased the
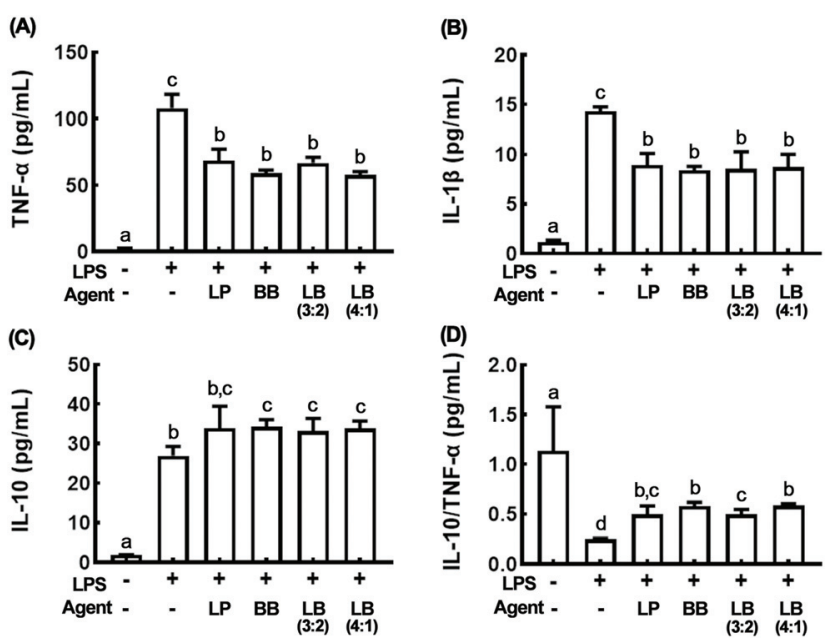

Fig. 1 Effects of NK151 and NK175 on the LPS-induced TNF- $\alpha$, IL-1 and IL-10 expression in macrophage cells. Effects on TNF- $\alpha$ (A), IL-1 $\beta$ (B), and IL-10 expression (C) and the IL-10 to TNF- $\alpha$ expression ratio (D). Macrophage cells $\left(1 \times 10^{6} \mathrm{CFU} \mathrm{ml} \mathrm{m}^{-1}\right)$ isolated from the peritoneal cavity were incubated with NK151 (LP), NK175 (BB), and their $(3: 2)$ and $(4: 1)$

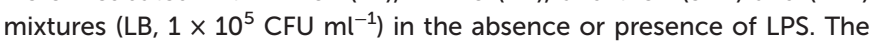
control group (CON) was treated with saline instead of LPS. Data values are described as mean \pm SD $(n=4)$. Means with the same letters are not significantly different $(p<0.05)$.
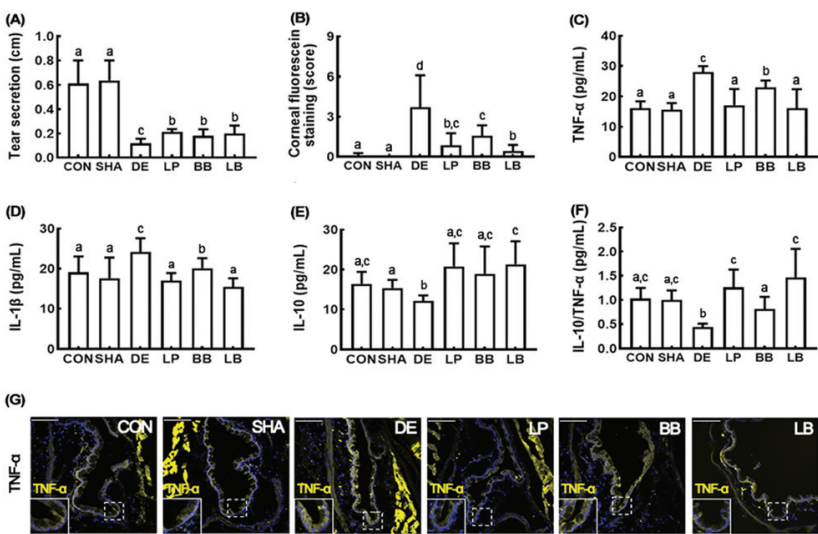

Fig. 2 NK151 and NK175 alleviated ELA-induced dry eye in C57BL/ 6 mice. (A) Effects on tear secretion. (B) Effects on corneal fluoresceinstaining score. Effects on the TNF- $\alpha$ (C), IL-1 $\beta$ (D), and IL-10 expression (E) and IL-10 to TNF- $\alpha$ expression ratio (F), assessed by ELISA. (G) Effects on the TNF- $\alpha$ expression in the conjunctiva, assessed by immunofluorescence assay. NK151 (LP), NK175 (BB), and their (3:2) mixture (LB, $5 \times$ $10^{9}$ CFU per mouse per day) were orally gavaged daily for 10 days from $25 \mathrm{~h}$ after the exposure to ELA (exorbital lacrimal gland excision and 1\% atropine/0.1\% benzalkonium chloride solution treatment). Normal control mice (CON), sham mice (SHA), and mice exposed to ELA (DE) were treated with the vehicle instead of probiotics. Data values are described as mean \pm SD $(n=7)$. Means with the same letters are not significantly different $(p<0.05)$.

ELA-induced corneal fluorescein-staining score. Of these, NKM most potently suppressed the corneal fluorescein-staining score to $0.4 \pm 0.5$, followed by NK151 and NK175.

Next, we examined the effects of NK151, NK175, or NKM on the IL-1 $\beta$, IL-10, and TNF- $\alpha$ expression in the conjunctiva of ELA-exposed mice using ELISA (Fig. 2C-E). ELA exposure significantly increased the IL-1 $\beta$ and TNF- $\alpha$ expression, while the IL-10 expression was decreased. However, oral gavage of NK151, NK175, or NKM significantly suppressed the IL-1 $\beta$ and TNF- $\alpha$ expression and induced the IL-10 expression. In particular, they increased the ratio of IL-10 to TNF- $\alpha$ expression (Fig. $2 \mathrm{~F}$ ). Of these, NKM increased the ratio of IL-10 to TNF- $\alpha$ expression most potently. Assessed by the immunohistochemistry assay, NK151, NK175, or NKM suppressed the ELA-induced TNF- $\alpha$ expression in the conjunctiva of ELA-exposed mice.

\subsection{NK151, NK175, and NKM alleviated ELA-induced colitis in mice}

We also examined the effects of NK151, NK175, or NKM on the myeloperoxidase activity and IL-1 $\beta$, IL-10, and TNF- $\alpha$ expression in the colon of ELA-exposed mice (Fig. 3). Exposure to ELA increased the myeloperoxidase activity, IL-1 $\beta$ and TNF- $\alpha$ expression and NF- $\mathrm{KB}^{+} / \mathrm{CD} 11 \mathrm{c}^{+}$cell population in the colon of mice, while the IL-10 expression was decreased (Fig. 3A-D and F). However, oral gavage of NK151, NK175, or NKM suppressed the IL- $1 \beta$ and TNF- $\alpha$ expression and NF- $\mathrm{KB}^{+} / \mathrm{CD} 11 \mathrm{c}^{+}$cell population and induced the IL-10 expression. In particular, they also increased the ratio of IL-10 to TNF- $\alpha$ expression in the colon (Fig. 3E). 

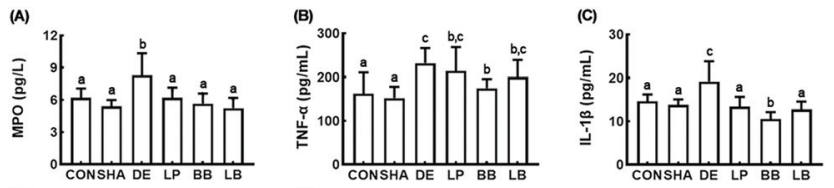

(D)

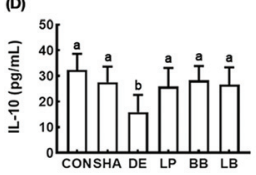

(E)

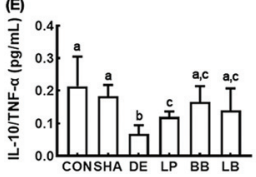

(F)

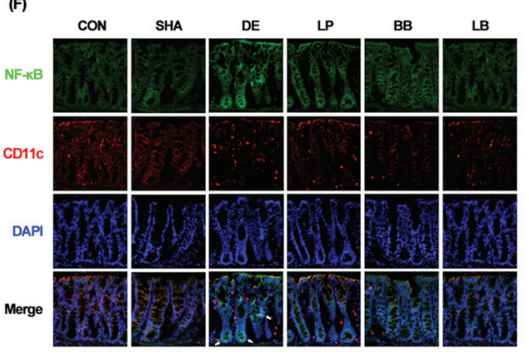

Fig. 3 NK151 and NK175 alleviated ELA-induced colitis in C57BL/ 6 mice. Effects on the myeloperoxidase (MPO) activity (A), TNF- $\alpha$ (B), IL-1 $\beta(C)$, and IL-10 expression (D), IL-10 to TNF- $\alpha$ expression ratio (E), and NF- $\mathrm{KB}^{+} / \mathrm{CD} 11 \mathrm{c}^{+}$cell population (F) in the colon. NK151 (LP), NK175 (BB), and their $(3: 2)$ mixture (LB, $5 \times 10^{9} \mathrm{CFU}$ per mouse per day) were orally gavaged daily for 10 days from $25 \mathrm{~h}$ after the exposure to ELA (exorbital lacrimal gland excision and 1\% atropine/0.1\% benzalkonium chloride solution treatment). Normal control mice (CON), sham mice (SHA), and mice exposed to ELA (DE) were treated with the vehicle instead of probiotics. Data values are described as mean \pm SD $(n=7)$. Means with the same letters are not significantly different $(p<0.05)$.

\subsection{NK151, NK175, and NKM alleviated ELA-induced gut} dysbiosis in mice

Exposure to ELA caused colitis with dry eye in mice. Therefore, we examined the effects of NK151, NK175, or NKM on the gut microbiota composition in mice (Fig. 4). ELA exposure significantly reduced the $\alpha$-diversity (OUT richness) compared to that of sham mice (Fig. 4A). However, oral gavage of NK175 most potently increased ELA-induced $\alpha$-diversity reduction to that of the control group, followed by NKM and NK151. Exposure to ELA also significantly shifted the $\beta$-diversity (PCoA) (Fig. 4B). Oral gavage of NK151 and NK175 partially restored the ELAshifted $\beta$-diversity to that of the control group. ELA exposure increased Actinobacteria, Verrucomicrobia, and Proteobacteria populations and weakly, but not significantly, decreased Firmicutes and Bacteroidetes populations at the phylum level (Fig. 4C and ESI Tables S3-S6†). However, NK151 and NK175 reduced ELA-induced Verrucomicrobia and Actinobacteria populations. Furthermore, oral gavage of NK151, NK175, or NKM reduced ELA-induced Bacteroidaceae, Akkemansiaceae, and AC160630_f populations at the family level, PAC000664_g and PAC001092_g, populations at the genus level, and Bacteroides acidifaciens group, PAC001982_s, PAC002482_S group, and AB606242-s populations at the species level (Fig. 4D). Their treatments increased the ELA-suppressed Lactobacillaceae and Muribaculaceae populations at the family level, Lactobacillus, Prevotellaceae_uc, PAC001068_g, PAC000186_g, KE159538_g, and PAC000198_g populations at the genus level, and Lactobacillus murinus group, Lactobacillus_uc, PAC001068_g_uc, and PAC001070_s group populations at the species level.
To understand what kinds of gut microbiota are associated with the therapeutic effects of NK151 and/or NK175 in mice with ELA-induced dry eye, we analyzed the correlation coefficient between the tear secretion amount and gut microbiota in mice treated with and without NK151, NK175, or NKM (Fig. 4E). Lactobacillaceae $(R=0.473, p=0.002)$, Lactobacillaceae_uc $(R=0.435, p=0.004)$, Lactobacillus $(R=$ $0.470, p=0.002)$, Ruminococcus_uc $(R=0.528, p=0.001)$, Lactobacillus murinus $(R=0.407, p=0.008)$, Lactobacillus reuteri $(R=0.107, p=0.501)$, and Ruminococcus_uc $(R=109, p=0.491)$ showed positive correlation with the tear secretion amount. However, Bacteroidaceae $(R=-0.345, p=0.025)$, Desulfovibrionaceae $(R=-0.477, p=0.001)$, Muribaculaceae $(R$ $=-0.198, p=0.208)$, Pseudoflavonifractor $(R=-0.615, p<$ $0.001)$, Muribaculaceae_uc $(R=-0.425, p=0.005)$, Bacteroides $(R=-0.348, p=0.024)$, and PA001061_S $(R=-0.252, p=0.108)$ showed negative correlation.

\subsection{NKm alleviated ELA-induced dry eye and colitis in mice}

To confirm the ameliorating effect of the NK151 and NK175 mixture against dry eye and understand the species/ strain specificity and mixing ratio in therapeutic effects, we examined the effect of NKm, the NK151 and NK175 $(4: 1)$ mixture, in BALB/c mice with ELA-induced dry eye (Fig. 5 and ESI Table $\mathrm{S} 2 \dagger)$. Treatment with ELA significantly reduced tear secretion to $28.9 \%$ of control mice after the final exposure to ELA $(p<0.05)$ (Fig. 5A). However, oral gavage of NKm significantly increased tear secretion to $40.6 \%$ of sham mice in ELAexposed mice after the final treatment with NKm. Oral gavage of NKm decreased ELA-induced corneal fluorescein-staining and lissamine green-staining scores (Fig. 5B and C). Oral gavage of NKm significantly suppressed the ELA-induced IL-1 $\beta$ and TNF- $\alpha$ expression and induced ELA-suppressed IL-10 expression using ELISA (Fig. 5D-F). Its treatment also increased the ratio of IL-10 to TNF- $\alpha$ expression (Fig. 5G). Assessed by the immunohistochemistry assay, NKm suppressed ELA-induced TNF- $\alpha$ expression and increased the number of goblet cells in the conjunctiva of ELA-exposed mice (Fig. 5H and I). Furthermore, NKm alleviated ELA-induced blinking and meibomian gland dysfunction (Fig. 5J and $\mathrm{K}$ ). Oral gavage of NKm significantly decreased ELA-induced IL-1 $\beta$ and $\mathrm{TNF}-\alpha$ expression and $\mathrm{NF}-\mathrm{KB}^{+} / \mathrm{CD} 11 \mathrm{c}^{+}$cell population and induced ELA-suppressed IL-10 expression in the colon: it increased the ratio of IL-10 to TNF- $\alpha$ expression (ESI Fig. S3†).

\section{Discussion}

DED is accompanied by corneal epithelial damage, ocular surface inflammation, and conjunctival goblet cell loss. ${ }^{2,3}$ Patients with DED exhibit gut dysbiosis. ${ }^{16,19}$ For example, patients with DED had a higher abundance of Veillonella and a lower abundance of Subdoligranulum in the gut microbiota composition compared to that of healthy individuals. ${ }^{19}$ Patients with Sjögren's syndrome, which exhibit dry eye, had a higher abundance of Bacteroidetes and a lower abundance of 


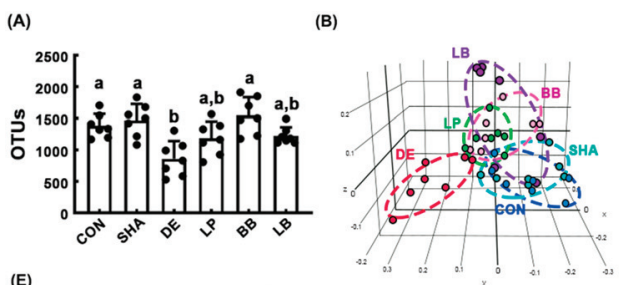

$\begin{array}{lll}\text { (C) Phylum } \quad \text { (E)Family } & \\ \end{array}$
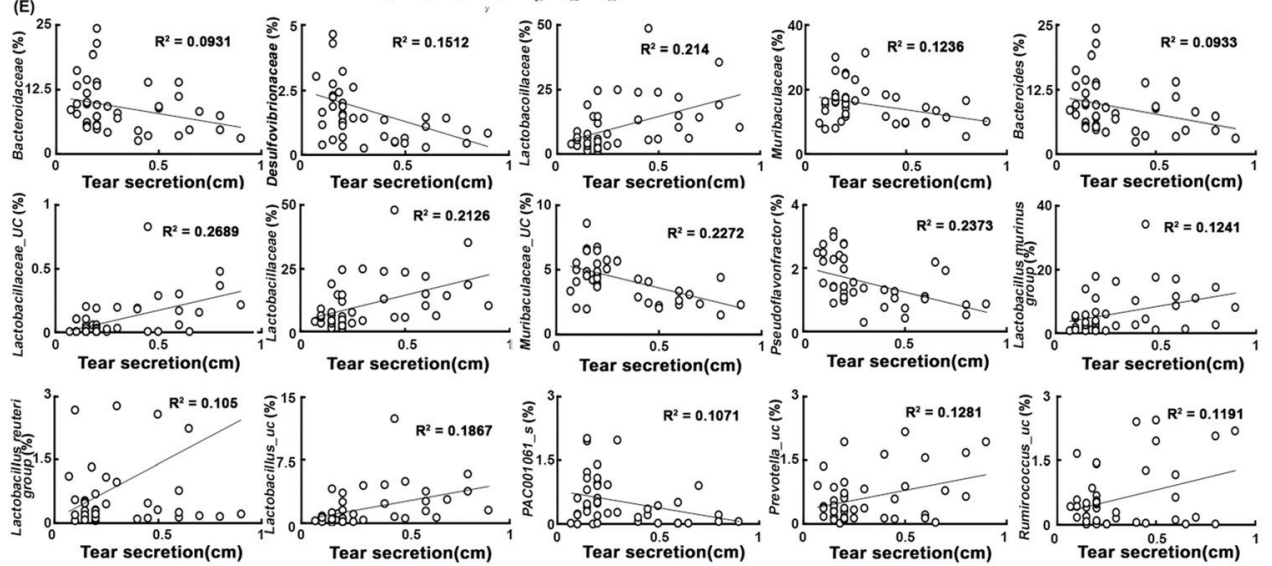

Fig. 4 Effects of NK151 and NK151 on the gut microbiota composition in C57BL/6 mice with ELA-induced dry eye. Effects on the $\alpha$-diversity (A, operational taxonomic units [OTUs]) and $\beta$-diversity (B, principal coordinate analysis [PCoA] plot based on Jansen-Shannon). Effects on the gut bacteria composition at the phylum (C) and family levels (D). (E) The correlation coefficient between the tear secretion amount and gut microbiota. NK151 (LP), NK175 (BB), and their (3:2) mixture [LB (3:2), $5 \times 10^{9}$ CFU per mouse per day] were orally gavaged daily for 10 days from $25 \mathrm{~h}$ after the exposure to ELA (exorbital lacrimal gland excision and $1 \%$ atropine/0.1\% benzalkonium chloride solution treatment). Normal control mice (CON), sham mice (SHA), and mice exposed to ELA (DE) were treated with the vehicle instead of probiotics. Data values are described as mean \pm SD $(n=7)$. The correlation coefficient was analyzed using Spearman's rho correlation analysis. Means with the same letters are not significantly different $(p<0.05)$.

Bifidobacterium compared to the healthy individual control. ${ }^{19}$ To evaluate therapeutic effects of drugs against DED, numerous animal models, such as NOD.B10.H2b mice, CD25 knockout mice, exorbital lacrimal gland-excised mice, intraorbital and exorbital lacrimal gland-excised mice, and desiccating stress/antibiotic-stimulated mice, have been developed. ${ }^{20,22,33-35}$ These animal models exhibit ocular and conjunctival inflammation and gut dysbiosis with dry eye. Although not sufficiently studied, these results suggest that gut dysbiosis may be closely associated with the occurrence and severity of DED. Therefore, alleviating gut dysbiosis may be beneficial for the therapy of DED.

In the present study, we found that exposure to ELA caused tear secretion reduction and goblet cell loss in the conjunctiva. Moreover, its exposure caused conjunctival inflammation: it increased the expression of proinflammatory cytokines TNF- $\alpha$ and IL-1 $\beta$ and their expression ratio to anti-inflammatory cytokine IL-10 in the conjunctiva, which was a negative correlation with the number of goblet cells. Moreover, conjunctival inflammation reduces the goblet cell loss. ${ }^{7,36}$ Goblet cells secrete antibacterial proteins. ${ }^{37}$ Oral administration of NK151, NK175, and their mixtures NKM and NKm alleviated ELAinduced tear secretion reduction and corneal epithelial damage in C57BL/6 and BALB/c mice with ELA-induced dry eye. Their treatments suppressed IL- $1 \beta$ and TNF- $\alpha$ expression and increased IL-10 expression in the conjunctiva. In particular, they increased the ratio of IL-10 to TNF- $\alpha$ expression. The stimulation of TNF- $\alpha$ and IL-1 $\beta$ induces the differentiation of Th0 into Th1 cells and polarization of resident macrophages into M1 macrophages. ${ }^{38,39}$ The stimulation of IL-10 induces the differentiation of Th0 into Treg cells and polarization of M1 into M2 macrophages. ${ }^{40,41}$ These results suggest that exposure to ELA can cause dry eye with ocular inflammation and NK151 and/or NK175 can alleviate dry eye and ocular and conjunctival inflammation by regulating Th1 and Treg cell differentiation and macrophage polarization through the expression of pro-inflammatory and anti-inflammatory cytokines. Moreover, oral administration of NK151 and/or NK175 increased ELA-suppressed goblet cell loss. These results suggest that NK151 and NK175 may alleviate conjunctival inflammation by increasing the secretion of antibacterial proteins from goblet cells.

Exposure to ELA also increased the myeloperoxidase activity, TNF- $\alpha$ and IL- $1 \beta$ expression, and NF- $\mathrm{KB}^{+} / \mathrm{CD} 11 \mathrm{c}^{+}$cell population and decreased the IL-10 expression and IL-10 to TNF- $\alpha$ expression ratio, resulting in colitis. Oral gavage of NK151 and/or NK175 ELA alleviated ELA-induced colitis. These results suggest that oral administration of NK151 and/or NK175 may alleviate ocular/conjunctival inflammation with colitis by regulating the gut immune system including Treg cell differentiation and NF-אB activation.

Sjögren's syndrome is an autoimmune disease that weakens the body's moisture-secreting glands such as the lacrimal and salivary glands. ${ }^{40}$ The gut microbiota composition of 

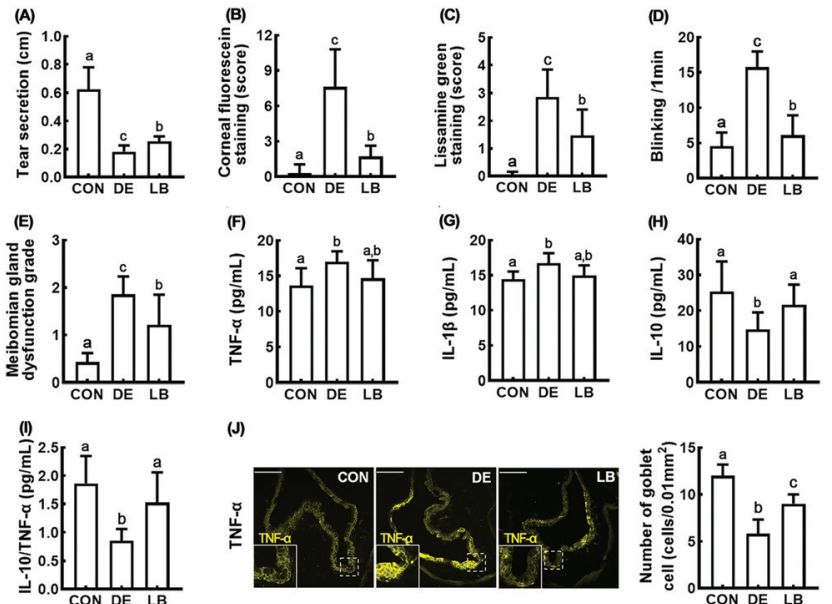

(J)
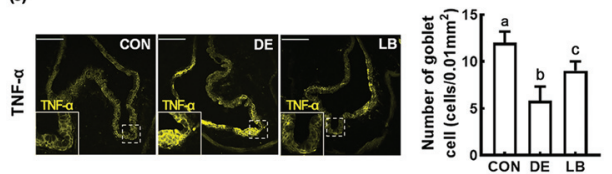

Fig. 5 The mixture of NK151 and NK175 (4:1), NKm, alleviated ELAinduced dry eye in BALB/c mice. (A) Effects on tear secretion. Effects on corneal fluorescein-staining (B) and lissamine green-staining scores (C). Effects on the TNF- $\alpha$ (D), IL-1 $\beta$ (E), and IL-10 expression (F) and IL-10 to TNF- $\alpha$ expression ratio (G), assessed by ELISA. Effects on the goblet cell counts $(\mathrm{H})$ and TNF- $\alpha$ expression (I) in the conjunctiva, assessed by immunofluorescence assay. Effects on the blinking (J) and meibomian gland dysfunction grade (K). NKm (LB, the NK151 and NK175 [4:1] mixture, $5 \times 10^{9} \mathrm{CFU}$ per mouse per day) was orally gavaged daily for 10 days from $25 \mathrm{~h}$ after the exposure to ELA (exorbital lacrimal gland excision and $1 \%$ atropine $/ 0.1 \%$ benzalkonium chloride solution treatment). Normal control mice (CON) and mice exposed to ELA (DE) were treated with the vehicle instead of probiotics. Data values are described as mean $\pm \mathrm{SD}(n=7)$. Means with the same letters are not significantly different $(p<0.05)$.

patients with Sjögren's syndrome was significantly different from that of healthy individuals. ${ }^{19}$ Furthermore, Sjögren's syndrome exhibits reduced diversity in the gut microbiota. However, the gut microbiota composition of patients with DED is also different from those of patients with Sjögren's syndrome and healthy individuals. Nevertheless, the gut microbiota composition and their byproducts such as endotoxins and SCFAs directly correlated with DED have not been sufficiently studied.

In the present study, we found that the reduction of tear secretion by exposure to ELA caused the alteration of the gut microbiota composition in mice: it reduced $\alpha$-diversity and shifted $\beta$-diversity. The results suggest that oral gavaged NK151 and/or NK175 may suppress ELA-induced gut microbiota growth. Among the gut bacteria, ELA exposure increased Actinobacteria, Verrucomicrobia, and Proteobacteria populations and decreased Firmicutes and Bacteroidetes populations. However, Moon et al. reported that the Bacteroidetes population was higher in the feces of patients with Sjögren's syndrome than in those of healthy volunteers, while the Bifidobacterium population was lower. ${ }^{19}$ We also found that Lactobacillaceae including Lactobacillaceae_uc, Lactobacillus murinus and Lactobacillus reuteri and Ruminococcus sp. including Ruminococcus_uc showed positive correlation with the tear secretion amount, while Bacteroidaceae Desulfovibrionaceae, and Muribaculaceae showed negative correlation. However,
NK151 and/or NK175 reduced ELA-induced Verrucomicrobia and Actinobacteria populations. Furthermore, oral gavage of NK151 and/or NK175 reduced the ELA-induced Bacteroidaceae, Akkemansiaceae, and AC160630_f populations and increased ELA-suppressed Lactobacillaceae and Muribaculaceae populations at the family level. Furthermore, they increased the ELAsuppressed populations of the Lactobacillus murinus group, Lactobacillus_uc, PAC001068_g_uc, and the PAC001070_s group at the species level. However, NK175, which is Bifidobacterium bifidum, did not affect the Lactobacillaceae population. IRT-5, a probiotic mixture, alleviates dry eye, suppresses conjunctival IL-1 $\beta$ expression, and increases conjunctival IL-10 expression and fecal Lactobacillus helveticus and Lactobacillus hamsteri populations in antibiotic-treated NOD.B10.H2b mice. ${ }^{22}$ Oral administration of a Bifidobacterial mixture with fructooligosaccharide (a symbiotic combination) alleviates dry eye syndrome in patients with DED. ${ }^{23}$ These results suggest that dry eye can cause ocular and conjunctival inflammation by altering the gut microbiota composition and their byproducts and the proliferation of gut Bifidobacteria and Lactobacilli may alleviate tear secretion. Although the exact mechanism needs to be further elucidated, NK151 and/or NK175 can alleviate dry eye with colitis by regulating the inflammatory response through the modulation of the gut microbiota composition.

\section{Conclusions}

Exposure to ELA caused tear secretion reduction, ocular/conjunctival inflammation, goblet cell loss, and gut microbiota alteration in mice. Oral administration of NK151 and/or NK175 alleviated ELA-induced dry eye with ocular/conjunctival inflammation and goblet cell loss by modulating the expression ratio of pro-inflammatory cytokines such as TNF- $\alpha$ and anti-inflammatory cytokines such as IL-10 and gut microbiota composition.

\section{Conflicts of interest}

The authors have declared no conflicts of interest.

\section{Acknowledgements}

This research was funded by the Medical Research Program through the National Research Foundation of Korea (NRF) funded by the Ministry of Science and ICT (NRF2017R1A5A2014768).

\section{References}

1 P. A. Rouen and M. L. White, Dry eye disease: Prevalence, assessment, and management, Home Healthcare Now, 2018, 36, 74-83. 
2 Q. Le, X. Cui, J. Xiang, L. Ge, L. Gong and J. Xu, Impact of conjunctivochalasis on visual quality of life: a community population survey, PLoS One, 2014, 9, e110821.

3 S. Jaiswal, L. Asper, J. Long, A. Lee, K. Harrison and B. Golebiowski, Ocular and visual discomfort associated with smartphones, tablets and computers: what we do and do not know, Clin. Exp. Optom., 2019, 102, 463-477.

4 I. K. Gipson, Age-related changes and diseases of the ocular surface and cornea, Invest. Ophthalmol. Visual Sci., 2013, 54, ORSF48-ORSF53.

5 P. Thulasi and A. R. Djalilian, Update in current diagnostics and therapeutics of dry eye disease, Ophthalmology, 2017, 124, S27-S33.

6 D. Zoukhri, Effect of inflammation on lacrimal gland function, Exp. Eye Res., 2006, 82, 885-898.

7 K. S. Kunert, A. S. Tisdale and I. K. Gipson, Goblet cell numbers and epithelial proliferation in the conjunctiva of patients with dry eye syndrome treated with cyclosporine, Arch. Ophthalmol., 2002, 120, 330-337.

8 J. Shimazaki, Definition and diagnostic criteria of dry eye disease: Historical overview and future directions, Invest. Ophthalmol. Visual Sci., 2018, 59, DES7-DES12.

9 S. Shimmura, M. Ono, K. Shinozaki, I. Toda, E. Takamura, Y. Mashima and K. Tsubota, Sodium hyaluronate eyedrops in the treatment of dry eyes, Br. J. Ophthalmol., 1995, 79, 1007-1011.

10 L. L. Marshall and J. M. Roach, Treatment of dry eye disease, Consult. Pharm., 2016, 31, 96-106.

11 H. N. Oh, C. E. Kim, J. H. Lee and J. W. Yang, Effects of quercetin in a mouse model of experimental dry eye, Cornea, 2015, 34, 1130-1136.

12 F. Sommer and F. Bäckhed, The gut microbiota-masters of host development and physiology, Nat. Rev. Microbiol., 2013, 11, 227-238.

13 S. M. Jandhyala, R. Talukdar, C. Subramanyam, H. Vuyyuru, M. Sasikala and D. N. Reddy, Role of the normal gut microbiota, World J. Gastroenterol., 2015, 21, 8787-8803.

14 H. J. Harmsen and M. C. de Goffau, The human gut microbiota, Adv. Exp. Med. Biol., 2016, 902, 95-108.

15 T. G. Dinan and J. F. Cryan, Brain-gut-microbiota axis and mental health, Psychosom. Med., 2017, 79, 920-926.

16 K. M. Cavuoto, S. Banerjee and A. Galor, Relationship between the microbiome and ocular health, Ocul. Surf., 2019, 17, 384-392.

17 P. Lin, The role of the intestinal microbiome in ocular inflammatory disease, Curr. Opin. Ophthalmol., 2018, 29, 261-266.

18 C. Tsigalou, E. Stavropoulou and E. Bezirtzoglou, Current insights in microbiome shifts in sjogren's syndrome and possible therapeutic interventions, Front. Immunol., 2018, 9, 1106.

19 J. Moon, S. H. Choi, C. H. Yoon and M. K. Kim, Gut dysbiosis is prevailing in Sjögren's syndrome and is related to dry eye severity, PLoS One, 2020, 15, e0229029.

20 C. S. de Paiva, D. B. Jones, M. E. Stern, F. Bian, Q. L. Moore, S. Corbiere, C. F. Streckfus, D. S. Hutchinson,
N. J. Ajami, J. F. Petrosino and S. C. Pflugfelder, Altered mucosal microbiome diversity and disease severity in sjögren syndrome, Sci. Rep., 2016, 6, 23561.

21 M. Zaheer, C. Wang, F. Bian, Z. Yu, H. Hernandez, R. G. de Souza, K. T. Simmons, D. Schady, A. G. Swennes, S. C. Pflugfelder, R. A. Britton and C. S. de Paiva, Protective role of commensal bacteria in Sjögren syndrome, J. Autoimmun., 2018, 93, 45-56.

22 H. Choi, J. W. Oh, J. S. Ryu, H. M. Kim, S. H. Im, K. P. Kim and M. K. Kim, IRT5 probiotics changes immune modulatory protein expression in the extraorbital lacrimal glands of an autoimmune dry eye mouse mode, Invest. Ophthalmol. Visual Sci., 2020, 61, 42.

23 G. Chisari, E. M. Chisari, A. Francaviglia and C. G. Chisari, The mixture of bifidobacterium associated with fructooligosaccharides reduces the damage of the ocular surface, Clin. Ter., 2017, 168, e181-e185.

24 H. M. Jang, S. K. Han, J. K. Kim, S. J. Oh, H. B. Jang and D. H. Kim, Lactobacillus sakei alleviates high-fat-dietinduced obesity and anxiety in mice by inducing AMPK activation and SIRT1 expression and inhibiting gut microbiota-mediated NF-кB activation, Mol. Nutr. Food Res., 2019, 63, e1800978.

25 N. E. Mecum, D. Cyr, J. Malon, D. Demers, L. Cao and I. D. Meng, Evaluation of corneal damage after lacrimal gland excision in male and female mice, Invest. Ophthalmol. Visual Sci., 2019, 60, 3264-3274.

26 D. Dursun, M. Wang, D. Monroy, D. Q. Li, B. L. Lokeshwar, M. E. Stern and S. C. Pflugfelder, A mouse model of keratoconjunctivitis sicca, Invest. Ophthalmol. Visual Sci., 2002, 43, 632-638.

27 J. M. Yang, F. Li, Q. Liu, M. Rüedi, E. T. Wei, M. Lentsman, H. S. Lee, W. Choi, S. J. Kim and K. C. Yoon, A novel TRPM8 agonist relieves dry eye discomfort, $B M C$ Ophthalmol., 2017, 17, 101.

28 Z. Lin, X. Liu, T. Zhou, Y. Wang, L. Bai, H. He and Z. Liu, A mouse dry eye model induced by topical administration of benzalkonium chloride, Mol. Vision, 2011, 17, 257-264.

29 C. Simsek, T. Kojima, T. Nagata, M. Dogru and K. Tsubota, Changes in murine subbasal corneal nerves after scopolamine-induced dry eye stress exposure, Invest. Ophthalmol. Visual Sci., 2019, 60, 615-623.

30 Z. Y. Dong, M. Ying, J. Zheng, L. J. Hu, J. Y. Xie and Y. Ma, Evaluation of a rat meibomian gland dysfunction model induced by closure of meibomian gland orifices, Int. J. Ophthalmol., 2018, 11, 1077-1083.

31 H. Wu, Y. Wang, N. Dong, F. Yang, Z. Lin, X. Shang and C. Li, Meibomian gland dysfunction determines the severity of the dry eye conditions in visual display terminal workers, PLoS One, 2014, 9, e105575.

32 J. K. Kim, K. E. Lee, S. A. Lee, H. M. Jang and D. H. Kim, Interplay between human gut bacteria Escherichia coli and Lactobacillus mucosae in the occurrence of neuropsychiatric disorders in mice, Front. Immunol., 2020, 11, 273. 
33 C. S. Kim, K. Jo, I. S. Lee and J. Kim, Topical application of apricot kernel extract improves dry eye symptoms in a unilateral exorbital lacrimal gland excision mouse, Nutrients, 2016, 8, 750.

34 K. Shinomiya, M. Ueta and S. Kinoshita, A new dry eye mouse model produced by exorbital and intraorbital lacrimal gland excision, Sci. Rep., 2018, 8, 1483.

35 K. C. Yoon, K. Y. Ahn, W. Choi, Z. Li, J. S. Choi, S. H. Lee and S. H. Park, Tear production and ocular surface changes in experimental dry eye after elimination of desiccating stress, Invest. Ophthalmol. Visual Sci., 2011, 52, 7267-7273.

36 D. A. Dartt and S. Masli, Conjunctival epithelial and goblet cell function in chronic inflammation and ocular allergic inflammation, Curr. Opin. Allergy Clin. Immunol., 2014, 14, 464-470.
37 J. J. Nair and T. P. Singh, Sjogren's syndrome: Review of the aetiology, Pathophysiology \& Potential therapeutic interventions, J. Clin. Exp. Dent., 2017, 9, e584-e589.

38 C. Atri, F. Z. Guerfali and D. Laouini, Role of human macrophage polarization in inflammation during infectious diseases, Int. J. Mol. Sci., 2018, 19, 1801.

39 W. Strober and I. J. Fuss, Proinflammatory cytokines in the pathogenesis of inflammatory bowel diseases, Gastroenterology, 2011, 140, 1756-1767.

40 D. W. Melton, L. M. McManus, J. A. L. Gelfond and P. K. Shireman, Temporal phenotypic features distinguish polarized macrophages in vitro, Autoimmunity, 2015, 48, 161-176.

41 H. Groux and F. Cottrez, The complex role of interleukin-10 in autoimmunity, J. Autoimmun., 2003, 20, 281-285. 